\title{
Mechanical dilator sheath markedly damaged by fibrosis
}

\begin{abstract}
Transvenous lead extraction (TLE) procedures, despite the high success rate could esistate in life-threatening consequences. The operator experience is crucial for the procedural outcome and every procedure can hide unexpected difficulties as the one encountered in the case presented. During a double coil lead defibrillator extraction, with an apparently preserved maneuverability of the mechanical dilator, a further control of the sheath revealed its complete fracture with the risk of losing part of it inside the vein.
\end{abstract}

Keywords: transvenous lead extraction, mechanical dialtor sheath, fibrosis
Volume 9 Issue 4 - 2017

\author{
L Bontempi,' G Mitacchione, ${ }^{1,2}$ A Curnis' \\ 'Department of Cardiology, Spedali Civili Hospital, University of \\ Brescia, Italy \\ ${ }^{2}$ Department of Heart and Vessels, Ospedale di Circolo e \\ Fondazione Macchi, University of Insubria, Italy
}

Correspondence: G Mitacchione, Cardiology Department, Spedali Civili Hospital, University of Brescia, Piazzale Spedali Civili I, 25I23 Brescia, Italy, Tel 39339420 6452,

Email gianfrancomit@hotmail.com

Received: June 13, 2017 | Published: August 07, 2017

\section{Introduction}

The need of transvenous lead extraction (TLE) procedures are steadily increasing nowadays with an estimated 10 000-15 000 pacemaker and implantable cardioverter-defibrillator (ICD) leads extracted annually worldwide. This increate number of TLE procedure is surely related to to the extensive use of cardiovascular implantable electronic devices (CIED) ${ }^{1}$ and to the population ageing. Despite the high success rate and safety in TLE procedures, ${ }^{2,3}$ is well knows how these could cause life-threatening consequences.

The development of specialized tools able to disrupt more easily encapsulating fibrous tissue do not reduce the crucial importance of the operator experience to avoid procedural complications4 The choose of the extraction tools (docking stylets, mechanical sheats, or powered sheaths) and the approach prefered during the procedure (other than the original venous insertion site) depend by the operator's experience and patients' characteristics. During our long experience in such procedures, we learned how these represent always a new challenge that can hide unexpected difficulties as the one in the case presented.

\section{Case presentation}

An 80 years old male patient was scheduled in our center for a TLE procedure. The patient was affected by idiopathic dilated cardiomyopathy with severely reduced left ventricular ejection fracion (LVEF $35 \%$ ) and received a cardiac resynchronization therapy defibrillator (CRT-D) device in 2009 with a dual coil defibrillator lead, active fixation (Riata $8 \mathrm{Fr}$ - St. Jude Medical). The TLE procedure indication was related to defibrillator lead externalized conductor (Figure 1 - arrow) with consequent inappropriate shocks therapy experienced by the patient.

Previously the procedure the venography showed left subclavian vein patency with fibrosis along the lead (Figure 2).

The defibrillator lead fixation screw was completely retracted and a locking stylet was inserted inside the lead. The first tool used for the extraction was a $10 \mathrm{Fr}$ polypropylene dilator sheath (Cook Medical). The advancement of the dilator over the lead was done with a pushing movement (while pulling the lead) balanced by clockwise and counterclockwise rotation under continuous fluoroscopic controll.

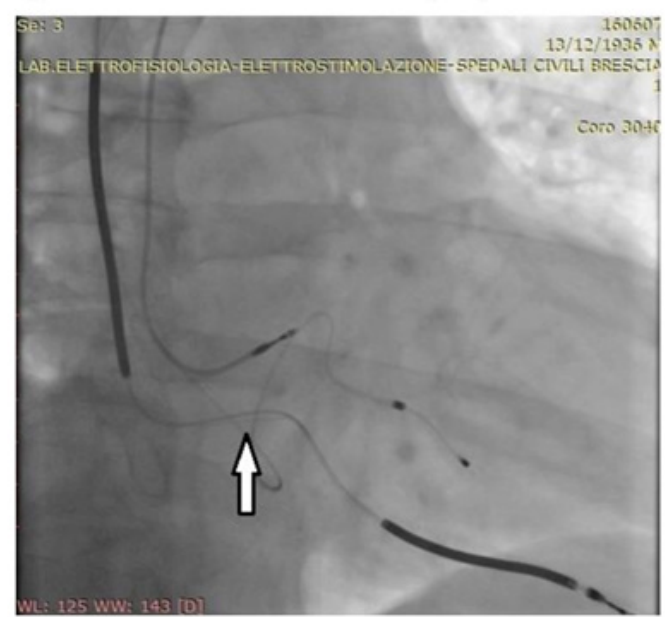

Figure I Defibrillator lead externalized conductor (arrow).

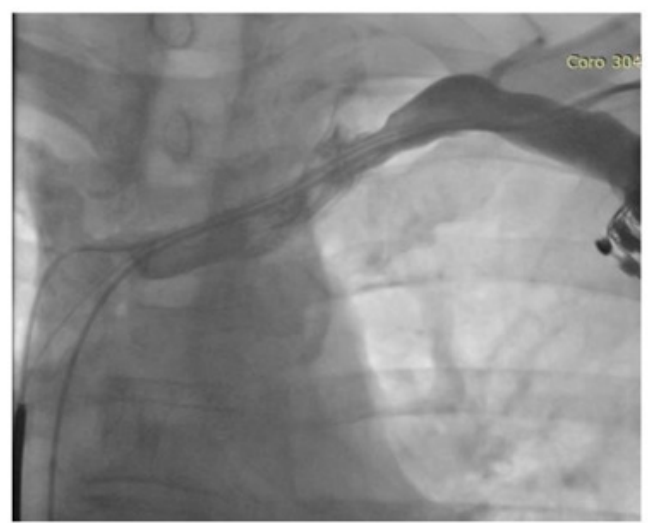

Figure $\mathbf{2}$ Venography showed left subclavian vein patency with fibrosis along the lead.

When in the proximal part of intavenous lead, the advancement of the dilator seemed able to disrupt the endovascular fibrosis which offered a moderate resistance to sheath advancement (Figure 3 Panel A) with apparent preserved rotational maneuverability. When 
removed, the sheath appeared markedly damaged (Figure 3 - Panel B) in the middle part. A further mechanical stress could have led to a complete sheath fracture with losing of its distal part inside the vein with dramatic consequences for the patient. A new polypropylene dilator sheath was then used and the procedure was successful accomplished.

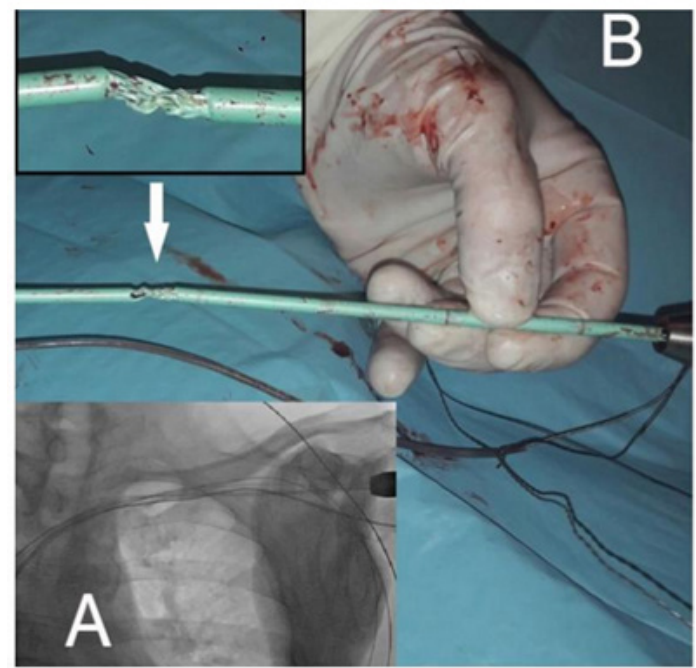

Figure 3 Panel A: Fluoroscopic control during dilator sheath advancement. Panel B: Dilator sheath dameged.

\section{Discussion}

This case presented suggested thath durimg TLE procedures performer with machanical dilator sheath in place of strong fibrosis, a continuous dilator check integrity, is desiderable even if a rotational maneuverability seems preserved to avoid dilator rupture. Mechanical dilator sheath integrity has to be checked frequently during extraction procedures. Fluoroscopic control cannot exclude sheath ruptures. In case of hard fibrosis alternative extraction methods have to be considered.

\section{Conflicts of interest \& disclosure}

Antonio Curnisis a consultant of Boston Scientific, Medtronic, Biotronik, Abbott, LivaNova, Spectranetics. Luca Bontempi is a consultant of Boston Scientific, Medtronic, Biotronik, Abbott, LivaNova.

\section{Acknowledgments}

None.

\section{Funding}

None.

\section{References}

1. Hauser RG, Katsiyiannis WT, GornickCC, etal.Deaths and cardiovascular injuries due to device-assisted implantable cardioverter-defibrillator and pacemaker lead extraction. Europace. 2010;12(3):395-401.

2. Kennergren C, Bjurman C, Wiklund R, et al. A single-centre experience of over one thousand lead extractions. Europace. 2009;11(5):612-617.

3. Bongiorni MG, Soldati E, Zucchelli G, et al. Transvenous removal of pacing and implantable cardiac defibrillating leads using single sheath mechanical dilatation and multiple venous approaches: high success rate and safety in more than 2000 leads. Eur Heart J . 2008;29(23):2886-2893.

4. Smith HJ, Fearnot NE, Byrd CL, et al. Five-years experience with intravascular lead extraction. U.S. Lead Extraction Database. Pacing Clin Electrophysiol. 1994;17(11 Pt 2):2016-2020. 